\title{
Prognostic effect of cytoreductive nephrectomy in synchronous metastatic renal cell carcinoma: a comparative study using inverse probability of treatment weighting
}

Tobias Klatte ${ }^{1}$, Kate Fife ${ }^{2}$, Sarah J. Welsh ${ }^{2}$, Manavi Sachdeva ${ }^{2}$, James N. Armitage ${ }^{1}$, Tevita 'Aho ${ }^{1}$, Antony C. Riddick' ${ }^{1}$, Athena Matakidou ${ }^{3}$, Tim Eisen ${ }^{3}$, Grant D. Stewart ${ }^{1}$

${ }^{1}$ Department of Urology, Cambridge University Hospitals NHS Foundation Trust, Cambridge, UK, ${ }^{2}$ Department of Oncology, Cambridge University Hospitals NHS Foundation Trust, Cambridge, UK, ${ }^{3}$ Department of Oncology, Cambridge University Hospitals NHS Foundation Trust, Cambridge, UK and AstraZeneca, Cambridge, UK

References: 23

Tables: 3

Figures: 3

Corresponding author:

Tobias Klatte

Department of Urology

Addenbrooke's Hospital

Hills Road

Cambridge, CB2 0QQ, UK

Phone: +44 1223245151

Electronic address: tobias.klatte@addenbrookes.nhs.uk 


\section{ABSTRACT (219 words)}

Purpose: To test the hypothesis that cytoreductive nephrectomy (CN) improves overall survival (OS) of patients with synchronous metastatic renal cell carcinoma (mRCC), who subsequently receive targeted therapies (TT).

Methods: We identified 261 patients who received TT for synchronous mRCC with or without prior $\mathrm{CN}$. To achieve balance in baseline characteristics between groups, we used the inverse probability of treatment weighting (IPTW) method. We conducted OS analyses, including IPTW-adjusted Kaplan-Meier curves, Cox regression models, interaction term, landmark and sensitivity analyses.

Results: Of the 261 patients, 97 (37.2\%) received CN and 164 (62.8\%) did not. IPTW-adjusted analyses showed a statistically significant OS benefit for patients treated with $\mathrm{CN}(\mathrm{HR}=0.63,95 \% \mathrm{Cl} 0.46-0.83, P=0.0015)$. While there was no statistically significant difference in OS at 3 months $(P=0.97), 6$ months $(P=0.67)$, and 12 months $(P=0.11)$ from diagnosis, a benefit for the $\mathrm{CN}$ group was noted at 18 months $(P=0.005)$ and 24 months $(P=0.004)$. On interaction term analyses, the beneficial effect of $\mathrm{CN}$ increased with better performance status $(P=0.06)$, in women $(P=0.03)$, and in patients with thrombocytosis $(P=0.01)$.

Conclusions: IPTW-adjusted analysis of our patient cohort suggests that CN improves OS of patients with synchronous mRCC treated with TT. On the whole, the survival difference appears after 12 months. Specific subgroups may particularly benefit from $\mathrm{CN}$, and these subgroups warrant further investigation in prospective trials.

Keywords: nephrectomy; sunitinib; pazopanib; prognosis; survival 


\section{INTRODUCTION}

Cytoreductive nephrectomy $(\mathrm{CN})$ has been an integral part of a multimodal management concept of patients with synchronous metastatic renal cell carcinoma (mRCC) treated with cytokines, as two randomised controlled trials demonstrated a significant overall survival (OS) advantage before treatment with interferon-alphabased therapy [1-3]. Over the past decade, targeted therapies (TT) with VEGF receptor tyrosine kinase inhibitors (TKI) and mammalian target of rapamycin (mTOR) inhibitors have replaced cytokine treatment and are now the accepted standard of care $[4,5]$. Since the inception of these agents, both the role of upfront $\mathrm{CN}$ and the timing of $\mathrm{CN}$ have been questioned. Two randomised controlled trials (SURTIME, CARMENA) were initiated to investigate the role of $\mathrm{CN}$ in conjunction with sunitinib. Unfortunately, both trials suffered from significant recruitment problems [6]. Indeed, SURTIME was stopped early and did not show a difference in progression-free survival at 28 weeks [7], which may in part due to sample size and insufficient statistical power. Likewise, there were considerable challenges with patient recruitment in CARMENA, and this trial is expected to end 6 years later than originally anticipated. Furthermore, there are some concerns that CARMENA may only answer the question of whether both trial arms are equivalent rather than showing than showing that one arm is superior $[6,8]$. Taken together, there is at present no level $1 / 2$ evidence regarding the role of $C N$ for $\mathrm{mRCC}$ treated with TT.

While results from these randomised controlled trials are awaited, best evidence for clinical practice and hypotheses for future randomised trials are derived from retrospective observational studies. Indeed, multi-centric and registry data suggest that $\mathrm{CN}$ may be associated with a $40 \%$ to $55 \%$ relative improvement in OS [8-10]. Despite multivariable adjustment for measured confounders, a prevailing hypothesis 
for this large benefit is selection bias. This could randomly favour the $\mathrm{CN}$ arm, as these patients may be those in a better general condition or those of a more favourable prognostic group. However, there are novel statistical methods such as inverse probability of treatment weighting (IPTW) and sensitivity analyses without assumptions that improve adjustment for measured and unmeasured confounders and thus control for selection bias [11]. As such, we sought to compare OS between patients undergoing $\mathrm{CN}$ and no $\mathrm{CN}(\mathrm{NCN})$ for synchronous $\mathrm{mRCC}$ at our tertiary care centre using these modern statistical approaches.

\section{PATIENTS AND METHODS}

\section{Patient population}

For this retrospective single centre study, patients were identified from the prospectively maintained Cambridge Oncology Registry. All patients treated with TT (VEGFR-TKIs or mTOR inhibitors) for synchronous clear cell and non-clear cell mRCC between 2006 and 2017 were identified; those with hereditary RCC syndromes, concomitant malignant tumours other than RCC and those who underwent complete surgical resection of all metastatic sites were excluded, leaving 261 patients as the principal study cohort. The decision to recommend a $\mathrm{CN}$ was based on a multidisciplinary team discussion of oncologists and urologists. Multiple variables were taken into consideration, including performance status (PS), tumour volume, number and location of metastatic sites, age, co-morbidity, surgical operability, expected surgical morbidity, and prognostic group according to initial 
blood tests. Patients were reviewed in clinic and a joint decision between patient, oncologist and urologist was made on whether to proceed with $\mathrm{CN}$ prior to TT.

Data for this study included receipt of $\mathrm{CN}$, age, gender, metastatic sites, type of medical therapies and prognostic criteria at the time of diagnosis, i.e. World Health Organization (WHO) PS, albumin-corrected serum calcium, haemoglobin, neutrophil count, platelet count, and time from diagnosis to targeted therapy [12]. WHO PS was then converted to the categorical Karnofsky PS (KPS; i.e. KPS $\geq 80 \%$ for WHO PS 0 or $1, \mathrm{KPS}<80 \%$ for WHO PS 2-4). Laboratory tests were performed in the Addenbrooke's Hospital laboratory and values were standardised against the upper or lower limit of normal, as appropriate [13].

\section{Statistical analyses}

Temporal trends in the practice pattern of $\mathrm{CN}$ were evaluated using a piecewise regression approach that is implemented in the Joinpoint Regression Program (Version 4.1, National Cancer Institute, Bethesda, MD, United States). In this approach, the annual frequency of $\mathrm{CN}$ was modelled using a linear segmented regression function, with a log-transformed dependent variable. Changes in temporal trend of the use of $\mathrm{CN}$ are reported as percentage change.

IPTW-adjusted analyses were performed to account for differences in baseline characteristics between groups and thus for selection bias, as popularised by Seisen et al. [11]. In this method, each patient was weighted by the inverse probability of being in the $\mathrm{CN}$ versus NCN group, with the goal of balancing observed characteristics between the two groups. The probability (or propensity) of being in the two treatment groups was estimated from a logistic regression model that included 
variables that potentially impacted receipt of $\mathrm{CN}$, i.e. age, presence of lung metastasis, liver metastasis, bone metastasis, brain metastasis, lymph node metastasis, number of metastatic sites, histological subtype, KPS, anaemia, neutrophilia, hypercalcemia, thrombocytosis and year of diagnosis. Baseline characteristics were compared between groups pre- and post-weighting using the standardised differences approach, as opposed to $t$ tests and $X^{2}$ tests. In this quantitative method, significant imbalances in covariates are present if the standardised difference is $\geq 0.1$ (i.e. $\geq 10 \%$ ).

The primary study endpoint was OS, which was calculated from the date of diagnosis to death or last follow-up. IPTW-adjusted survivor functions were estimated with the Kaplan-Meier method, and overall mortality was compared between groups using Cox proportional hazards regression models and IPTW-adjusted log-rank tests. The proportional hazards assumption was tested with Schoenfeld tests and complementary log-log plots, and demonstrated that this assumption was not violated in our models. Exploratory analyses were performed to determine the heterogeneity of the $\mathrm{CN}$ effect according to baseline variables by testing interaction terms within the IPTW-adjusted Cox models.

In view of the fact that IPTW-weighting balances only measured confounders between groups, we performed sensitivity analyses without assumptions to assess the impact of unmeasured confounders [14]. According to the approach described by Ding and VanderWheele [14], magnitudes of the joint bounding factor were estimated for various combinations of the odds of receiving $\mathrm{CN}$ in the (OR $\mathrm{ON}-\mathrm{U})$ and the hazard of overall mortality (HROM-U) both in the presence of unmeasured confounders. All statistical analyses were performed with R 3.4.0 (The R Foundation for Statistical Computing, Vienna, Austria). Statistical significance was set at 0.05. 


\section{RESULTS}

\section{Baseline characteristics}

The overall cohort included 261 patients with synchronous mRCC, of whom 97 (37.2\%) underwent $\mathrm{CN}$ and $164(62.8 \%)$ did not. The proportion of patients who underwent $\mathrm{CN}$ decreased over the years, with a biannual change of $-11.5 \%(95 \% \mathrm{Cl}$ 19.7 to $-2.5, P<0.001$, Fig. 1). The most common first line TT were sunitinib $(N=158$, $60.5 \%)$ and pazopanib $(N=74,28.4 \%)$. One hundred and twelve patients $(42.9 \%)$ received advanced line treatments (>first line), of which 22 (8.4\%) included cabozantinib or nivolumab.

Unweighted and weighted baseline characteristics are presented in Table 1. In unweighted comparisons, both groups differed with respect to all analysed baseline variables except gender and presence of lung metastases. After IPTW adjustment, all standardised differences were $<0.1$, indicating that both groups were then comparable.

\section{Overall survival}

There were 206 deaths $(78.9 \%)$ during follow-up. The median follow-up for patients surviving was 14.6 months (IQR 7.1 to 24.3). In unadjusted analyses, overall mortality was reduced in relative terms by $54 \%$ in the $\mathrm{CN}$ group (HR $0.46,95 \% \mathrm{Cl}$ 0.34 to $0.62, P<0.001)$, with a median OS time of 25.6 months $(95 \% \mathrm{Cl} 23.3$ to 32.1$)$ versus 12.4 months (95\% Cl 10.3 to 15.0 , Fig. $2 \mathrm{~A})$. In IPTW-adjusted analyses, the OS difference was smaller but still statistically significant (HR $0.63,95 \% \mathrm{Cl} 0.46$ to $0.83, P=0.0015)$, with a median OS time of 20.9 months $(95 \% \mathrm{Cl} 18.5$ to 29.6$)$ versus 12.6 months (95\% $\mathrm{Cl} 11.4$ to 15.2$)$ (Fig. 2B). As demonstrated in the IPTW-adjusted 
Kaplan-Meier plot (Fig. 2B), there was no impact of $\mathrm{CN}$ on $\mathrm{OS}$ probabilities at 3 months (CN versus NCN: $95.3 \%$ versus $95.2 \%, P=0.97$ ), at 6 months ( $84.6 \%$ versus $81.2 \%, P=0.67)$, and 9 months $(71.3 \%$ versus $67.9 \%, P=0.70)$. A clinically relevant OS benefit in favour of the $\mathrm{CN}$ group first appeared after 12 months (65.9\% versus $51.9 \%, P=0.11)$ and was statistically significant at 18 months $(59.2 \%$ versus $34.0 \%$, $P=0.005)$ and 24 months $(44.2 \%$ versus $21.8 \%, P=0.004)$. The 3 -month landmark IPTW-adjusted analysis demonstrated little impact of immortal time bias on treatment effect (HR $0.64,95 \% \mathrm{Cl} 0.48$ to $0.86, P=0.004)$. At adjusted 12-months landmark analysis that considered only patients alive at that landmark point, $\mathrm{CN}$ was associated with a $44 \%$ decreased relative risk of death (HR $0.56,95 \% \mathrm{Cl} 0.37$ to $0.85, P=0.006)$.

Using interaction term analyses, we tested whether type of treatment ( $\mathrm{CN}$ versus NCN) interacted with baseline predictors of overall mortality. IPTW-adjusted HR are presented in Figure 3. In these analyses, the beneficial effect of $\mathrm{CN}$ increased in patients with better KPS $(P=0.06)$, in women $(P=0.03)$, and in patients with thrombocytosis $(P=0.01)$. The effect of $\mathrm{CN}$ did not differ according to the type of TT $(P=0.47)$.

\section{Sensitivity analysis}

Magnitudes of the joint bounding factor for different combinations of the treatmentconfounder association ORCN-U and the mortality-confounder association HRom-U are shown in Table 2. For insignificance (yellow) or the opposite effect of CN (red), ORCN$\cup$ and HROM-U would need to meet specific estimates. The odds of receiving $\mathrm{CN}$ in the presence of a given unmeasured confounder (e.g. small volume metastatic disease) 
would need to increase, while the overall mortality would need to decrease in the presence of the same confounder. In a second set of sensitivity analysis, the cohort was restricted to patients with clear cell subtype. Propensity scores were reestimated for this subset, and the final models showed comparable results to the initial analysis without altering any conclusion. In final set of sensitivity analysis, we fitted a multivariable Cox model with baseline and treatment variables, including the effect of advanced line treatment. In this analysis, the beneficial effect of $\mathrm{CN}$ was confirmed (HR 0.68, $P=0.043$, Table 3).

\section{DISCUSSION}

In this retrospective study, we evaluated the prognostic effect of $\mathrm{CN}$ in patients with synchronous mRCC that subsequently received TT. In our cohort, $\mathrm{CN}$ had a statistically significant effect on overall mortality. Patients with a good KPS, women and those with thrombocytosis may benefit from $\mathrm{CN}$ and could represent the target population for future randomised trials.

The observed benefit in overall mortality for patients treated with $\mathrm{CN}$ is in line with other retrospective studies [8, 9], although our HR was slightly more conservative. Recently, Petrelli et al. [15] attempted a meta-analysis of retrospective studies and reported a pooled HR of 0.46 . However, as data quality was generally limited in these non-randomised observational studies, data from the pooled analysis were of limited quality as well. Further analyses from our study suggest that the beneficial effect of $\mathrm{CN}$ on mortality increases with increasing incremental survival time. Indeed, we did not find a difference in OS in the first 12 months of survivorship. Comparably, in 
adjusted analyses by Heng et al. [9], there was no statistically significant difference for patients who lived $<3$ months, 6 and 12 months; whereas there was a survival advantage for those patients estimated to survive longer. In contrast, in a large retrospective study from the National Cancer Database [8], statistical significance was obtained earlier than 12 months, but the absolute benefit was only 0.7 to 1.8 months. Furthermore, this study included almost 13,000 patients, which enabled the detection of very small statistically significant differences that were not present in smaller studies. Despite providing the largest sample size to date, this study did not account for performance status, laboratory data, IMDC prognostic group, or type of $\mathrm{TT}$, as these data were not available in this registry.

This, however, does not mean that patients with an estimated survival of $<12$ months do not benefit from a $\mathrm{CN}$. First, $\mathrm{CN}$ has clear role in symptom palliation, i.e. in patients with intractable pain, bleeding, uncontrolled hypertension, and symptoms due to paraneoplastic syndromes. Second, our interaction term analysis demonstrated an oncological benefit for certain subgroups, such as patients with thrombocytosis. Although generally considered as a group with dismal prognosis [13], $\mathrm{CN}$ may improve OS in this subgroup even though it may still be less than 12 months. The absolute benefit is minor and has to be balanced against the period of hospitalization and postoperative recovery. Further, with the low number of patients and the fairly large confidence interval, this finding has to be treated with caution. It is possible that it represents a statistical artefact rather than a true clinically relevant association. Similar conclusions can be drawn for gender and KPS, which represent the other two variables showing a clinically relevant interaction with CN. KPS, however, was previously identified as a predictor of outcomes. In a study by Heng et al. [9], the HR for $\mathrm{CN}$ was 0.53 in patients with $\mathrm{KPS}>80$ and 0.70 for patients with 
KPS $<80$, although no testing for heterogeneity or interaction was performed. KPS may therefore be a critical factor that determines the effect of $\mathrm{CN}$, but further validation studies are necessary.

Propensity score methods are often used to remove the effects of measured confounders in observational studies, including propensity score matching, covariate adjustment using propensity scores, and IPTW. IPTW creates a synthetic sample in which the distribution of measured baseline covariates is independent of treatment assignment [16] and allows relatively unbiased estimates of average treatment effects [17]. Of note, the design phase of the study is separated from the analysis phase, which is more comparable to randomised experiments [18]. IPTW serves to weight all groups up to the full sample [19], which is certainly an advantage over traditional matched pairs analyses. In urological cancer research, IPTW-adjusted analyses have been popularised by Seisen et al. [11] Using a similar approach, we were able to calculate a $\mathrm{HR}$ of $0.63(95 \% \mathrm{Cl} 0.46$ to 0.83$)$ in favour of $\mathrm{CN}$, which provided us with a more conservative estimate than data from unweighted analyses ( $\mathrm{HR} 0.46,95 \% \mathrm{Cl} 0.34$ to 0.62 ). We contend that in the setting of mRCC with many possible confounders, a more conservative approach is preferable.

As propensity scores can only adjust for measured confounders, unmeasured confounding must be addressed using distinct sensitivity analyses. Groups may differ with regards to these unmeasured confounders, which may subsequently impact the outcome measure [20]. We used sensitivity analyses according to Ding and VanderWheele, which has the advantage of making no assumptions about the structure of the unmeasured confounder and to provide stronger conclusions than traditional Cornfield conditions [14]. We show that, under certain circumstances, an 
unmeasured confounder would render the effect of $\mathrm{CN}$ insignificant, if there are imbalances in the odds for receiving a CN (yellow and red area in Table 2).

The role of $\mathrm{CN}$ in the era of immuno-oncology is unclear at present. In the CheckMate 025 trial, more than $85 \%$ of patients had prior $\mathrm{CN}$, but the impact of $\mathrm{CN}$ on outcomes was not analysed [21]. Cytoreductive surgery may be an important cornerstone in the multidisciplinary management of these patients, comparable to patients who received cytokines. The current study showed an OS benefit if patients received nivolumab or cabozantinib in the advanced line setting (Table 3). Further, the analysis reinforced the concept that there are multiple baseline (liver and node metastasis, thrombocytosis, KPS) and treatment characteristics (advanced line treatment) that dictate $\mathrm{OS}$, and $\mathrm{CN}$ may be one of them. Due to low numbers of patients, it was not possible to analyse the role of $\mathrm{CN}$ in patients receiving nivolumab. As a first step, an analysis of clinical trial data of CheckMate 025 may provide a hypothesis, and IPTW adjustment may be used for this purpose.

In randomised controlled trials, the survival time is generally calculated from the date of randomisation, which is not available in retrospective observational studies. Interestingly, there has been no consensus on how to define this starting point. While some groups used the time from the initiation of first-line targeted therapy [9, 22], Hanna et al. [8] employed the date of diagnosis, and others did not specify the exact method. Using the date from starting first-line treatment gives an artificial survival advantage of weeks to months to the group of patients who did not undergo $\mathrm{CN}$, as the period of nephrectomy and subsequent recovery is not included. Therefore, the authors of the present manuscript used the time of diagnosis, which may provide a better estimate of patient survival and may be more close to the date of randomisation. 
The current study is limited by a single-institution experience and its retrospective design. Subgroup analyses were limited by sample size. For example, in contrast to clear cell $\mathrm{mRCC}$, the $95 \% \mathrm{Cl}$ of the HR for non-clear cell included 1.00 . However, the $P$-value for the interaction test insignificant, which could be related to statistical power problems. Larger studies focusing on non-clear cell mRCC should address the question if $\mathrm{CN}$ is beneficial in this subgroup. As this was an analysis of an oncology database, the overall number of patients diagnosed with $\mathrm{mRCC}$ and the number of patients who received $\mathrm{CN}$ and then did not receive $\mathrm{TT}$ because of $\mathrm{CN}$-related morbidity, progressive disease or other reasons is unknown. Specifically, CN-related morbidity may be substantial, but it was not possible to account for that. In the British Association of Urological Surgeons nephrectomy audit, the 30-day mortality was $1.8 \%, 24.1 \%$ required perioperative blood transfusion and $8 \%$ had postoperative complications of Clavien-Dindo grade $\geq$ III [23]. Finally, selection bias and unmeasured differences between groups are a major problem in every retrospective study dealing with complex diseases such as mRCC. Several baseline parameters such as primary tumour volume (i.e. measured by size, local T stage on imaging) are important parameters which likely affected management but were not analysed, as no imaging review was performed. We attempted to address selection bias by performing IPTW-adjusted analyses according to many baseline variables and unmeasured confounding by sensitivity analysis. Nonetheless, residual unmeasured confounding may have impacted the effect of $\mathrm{CN}$. We recognise the limitations of both statistical approaches and that they cannot replace randomisation. 


\section{CONCLUSIONS}

IPTW-adjusted analysis of our patient cohort suggests that CN improves OS of patients with synchronous $\mathrm{mRCC}$ treated with TT. On the whole, the survival difference appears after 12 months. Specific subgroups may benefit from CN, and these subgroups warrant further investigation in prospective trials. 


\section{AUTHORS' CONTRIBUTION}

T Klatte Project development, Data collection, Data analysis, Manuscript writing

K Fife Data collection and management, Manuscript editing

SJ Welsh Data collection and management, Manuscript editing

M Sachdeva Data collection

JN Armitage Data collection

T 'Aho Data collection

AC Riddick Data collection

A Matakidou Data collection, Manuscript editing

T Eisen Project development, Data management, Manuscript editing

GD Stewart Project development, Data management, Manuscript editing 


\section{COMPLIANCE WITH ETHICAL STANDARDS}

\section{Disclosure of potential conflicts of interest}

The authors declare that they have no conflict of interest.

\section{Research involving Human Participants and/or Animals}

1) Statement of human rights

Ethical approval: All procedures performed in studies involving human participants were in accordance with the ethical standards of the institutional and/or national research committee and with the 1964 Helsinki declaration and its later amendments or comparable ethical standards. For this type of study formal consent is not required.

2) Statement on the welfare of animals

This article does not contain any studies with animals performed by any of the authors.

\section{Informed consent}

For this type of study formal consent is not required. 


\section{REFERENCES}

1. Mickisch GH, Garin A, van Poppel H, et al (2001) Radical nephrectomy plus interferon-alfa-based immunotherapy compared with interferon alfa alone in metastatic renal-cell carcinoma: a randomised trial. Lancet 358:966-970

2. Flanigan RC, Salmon SE, Blumenstein BA, et al (2001) Nephrectomy followed by interferon alfa-2b compared with interferon alfa- $2 b$ alone for metastatic renalcell cancer. N Engl J Med 345:1655-1659

3. Rodriguez Faba O, Brookman-May SD, Linares E, et al (2017) Cytoreductive nephrectomy in patients with metastatic renal cell carcinoma in the era of targeted therapy: a bibliographic review. World J Urol. doi: 10.1007/s00345-0172072-y

4. Bedke J, Gauler T, Grünwald V, et al (2017) Systemic therapy in metastatic renal cell carcinoma. World J Urol 35:179-188

5. Rothermundt C, von Rappard J, Eisen T, et al (2017) Second-line treatment for metastatic clear cell renal cell cancer: experts' consensus algorithms. World $\mathrm{J}$ Urol 35:641-648

6. Stewart GD, Aitchison M, Bex A, et al (2017) Cytoreductive Nephrectomy in the Tyrosine Kinase Inhibitor Era: A Question That May Never Be Answered. Eur Urol 71:845-847

7. Bex A, Mulders P, Jewett MAS, et al (2017) LBA35 - Immediate versus deferred cytoreductive nephrectomy $(\mathrm{CN})$ in patients with synchronous metastatic renal cell carcinoma (mRCC) receiving sunitinib (EORTC 30073 SURTIME). Ann Oncol 28:v605-v649

8. Hanna N, Sun M, Meyer CP, et al (2016) Survival Analyses of Patients With Metastatic Renal Cancer Treated With Targeted Therapy With or Without Cytoreductive Nephrectomy: A National Cancer Data Base Study. J Clin Oncol 34:3267-3275

9. Heng DYC, Wells JC, Rini BI, et al (2014) Cytoreductive nephrectomy in patients with synchronous metastases from renal cell carcinoma: results from the International Metastatic Renal Cell Carcinoma Database Consortium. Eur Urol 66:704-710

10. Abern MR, Scosyrev E, Tsivian M, et al (2014) Survival of patients undergoing cytoreductive surgery for metastatic renal cell carcinoma in the targeted-therapy era. Anticancer Res 34:2405-2411

11. Seisen T, Krasnow RE, Bellmunt J, et al (2017) Effectiveness of Adjuvant Chemotherapy After Radical Nephroureterectomy for Locally Advanced and/or Positive Regional Lymph Node Upper Tract Urothelial Carcinoma. J Clin Oncol $35: 852-860$ 
12. Noe A, de Bruijn RE, Blank C, et al (2016) Comparison of pre-treatment MSKCC and IMDC prognostic risk models in patients with synchronous metastatic renal cell carcinoma treated in the era of targeted therapy. World J Urol 34:1067-1072

13. Heng DYC, Xie W, Regan MM, et al (2009) Prognostic factors for overall survival in patients with metastatic renal cell carcinoma treated with vascular endothelial growth factor-targeted agents: results from a large, multicenter study. J Clin Oncol 27:5794-5799

14. Ding P, VanderWeele TJ (2016) Sensitivity Analysis Without Assumptions. Epidemiology 27:368-377

15. Petrelli F, Coinu A, Vavassori I, et al (2016) Cytoreductive Nephrectomy in Metastatic Renal Cell Carcinoma Treated With Targeted Therapies: A Systematic Review With a Meta-Analysis. Clin Genitourin Cancer 14:465-472

16. Austin PC (2011) An Introduction to Propensity Score Methods for Reducing the Effects of Confounding in Observational Studies. Multivar Behav Res 46:399_ 424

17. Austin PC, Stuart EA (2015) Moving towards best practice when using inverse probability of treatment weighting (IPTW) using the propensity score to estimate causal treatment effects in observational studies. Stat Med 34:3661-3679

18. Rubin DB (2001) Using Propensity Scores to Help Design Observational Studies: Application to the Tobacco Litigation. Health Serv Outcomes Res Methodol 2:169-188

19. Stuart EA (2010) Matching methods for causal inference: A review and a look forward. Stat Sci Rev J Inst Math Stat 25:1-21

20. Liu W, Kuramoto SJ, Stuart EA (2013) An Introduction to Sensitivity Analysis for Unobserved Confounding in Non-Experimental Prevention Research. Prev Sci Off J Soc Prev Res 14:570-580

21. Motzer RJ, Escudier B, McDermott DF, et al (2015) Nivolumab versus Everolimus in Advanced Renal-Cell Carcinoma. N Engl J Med 373:1803-1813

22. de Groot S, Redekop WK, Sleijfer S, et al (2016) Survival in Patients With Primary Metastatic Renal Cell Carcinoma Treated With Sunitinib With or Without Previous Cytoreductive Nephrectomy: Results From a Population-based Registry. Urology 95:121-127

23. Jackson BL, Fowler S, Williams ST, British Association of Urological Surgeons (BAUS) - Section of Oncology (2015) Perioperative outcomes of cytoreductive nephrectomy in the UK in 2012. BJU Int 116:905-910 


\section{FIGURE LEGENDS}

Figure 1 Temporal trends in utilisation of cytoreductive nephrectomy in 261 patients with synchronous metastatic RCC treated at Cambridge University Hospitals. There was a significant decline in the use of cytoreductive nephrectomy between $2006 / 2007$ and $2016 / 2017$ by $11.5 \%$ every two years $(95 \% \mathrm{Cl}-19.7$ to $-2.5, P<0.001)$.

Figure 2 Unadjusted $(\mathrm{A})$ and inverse probability of treatment weightingadjusted (B) Kaplan-Meier estimates of overall survival in patients who underwent cytoreductive nephrectomy versus no cytoreductive nephrectomy for synchronous metastatic renal cell carcinoma.

Figure 3 Forest plot depicting inverse probability of treatment weightingadjusted hazard ratios of overall mortality of cytoreductive nephrectomy versus no cytoreductive nephrectomy according to baseline clinical variables. Due to small numbers, the subgroups of patients with brain metastasis and an interval to TT $>12$ months were not analysed. 


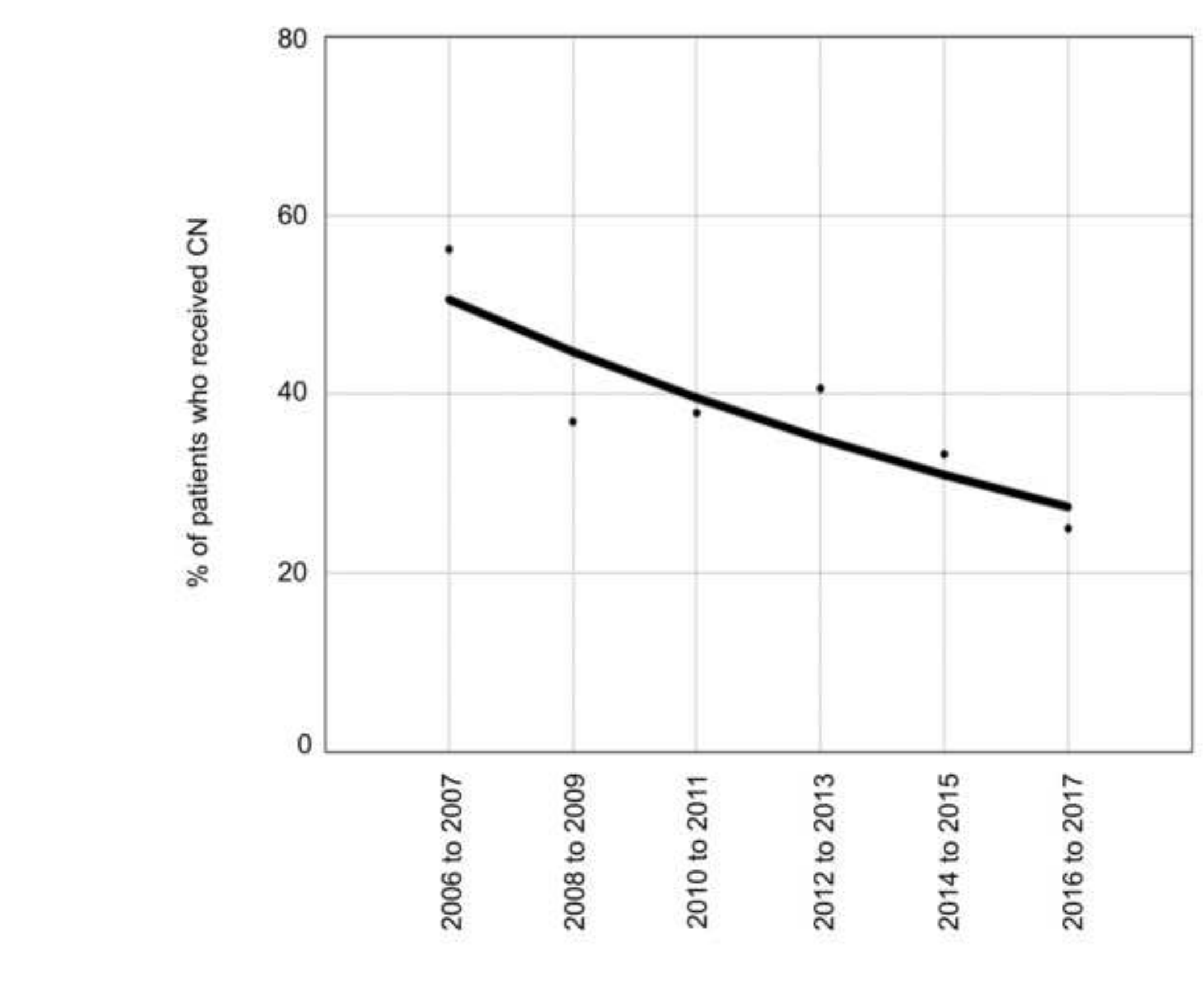

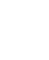

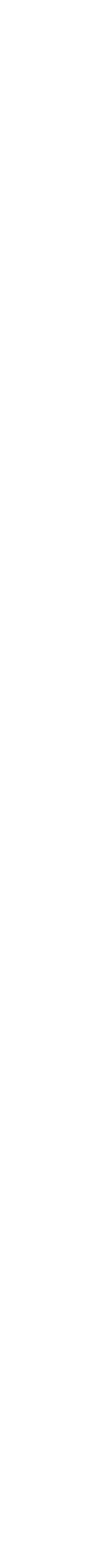

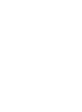

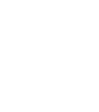
. 


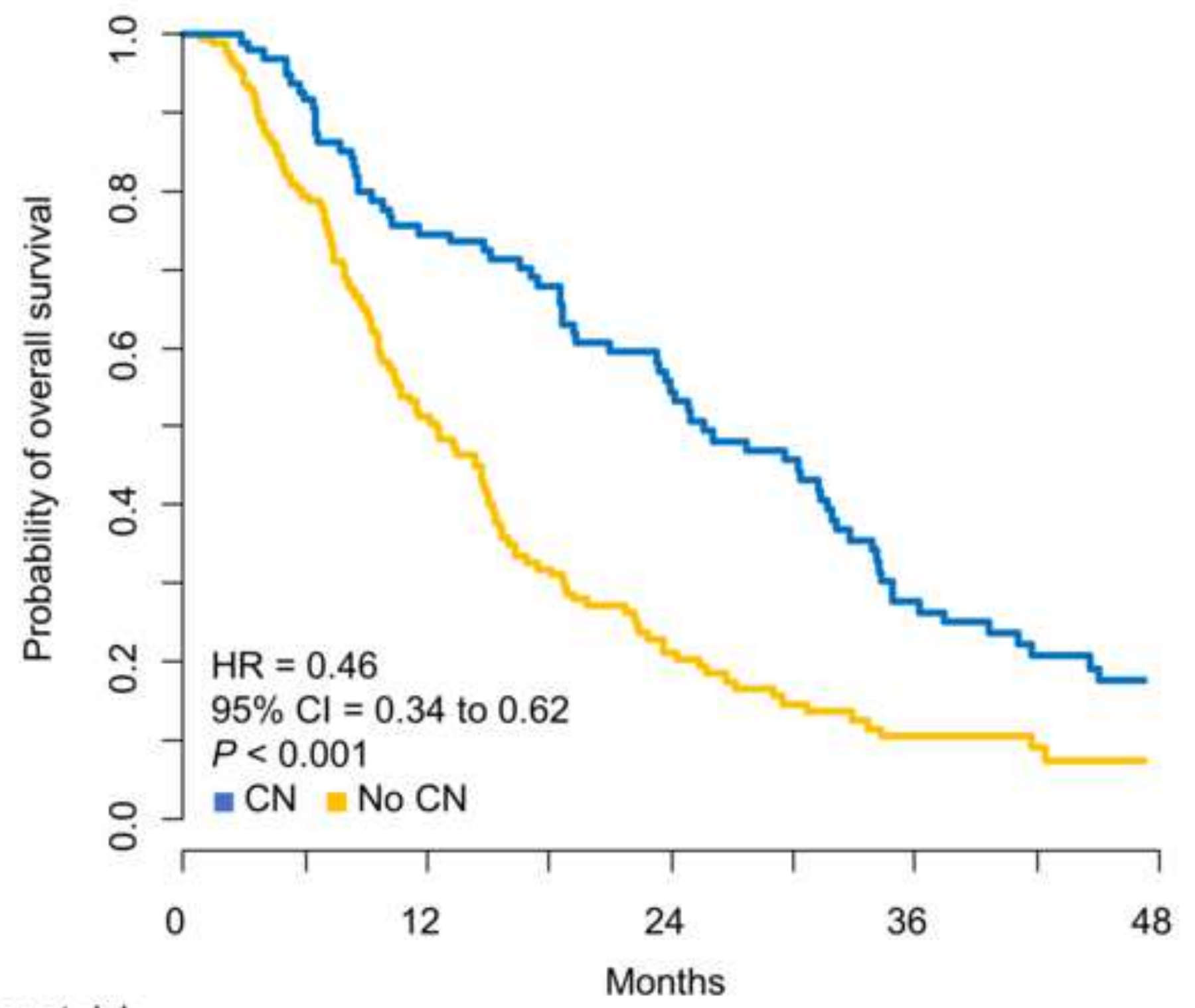

Number at risk

$\begin{array}{lccccc}\text { No CN } & 164 & 74 & 24 & 8 & 3 \\ \mathrm{CN} & 97 & 70 & 44 & 21 & 11\end{array}$




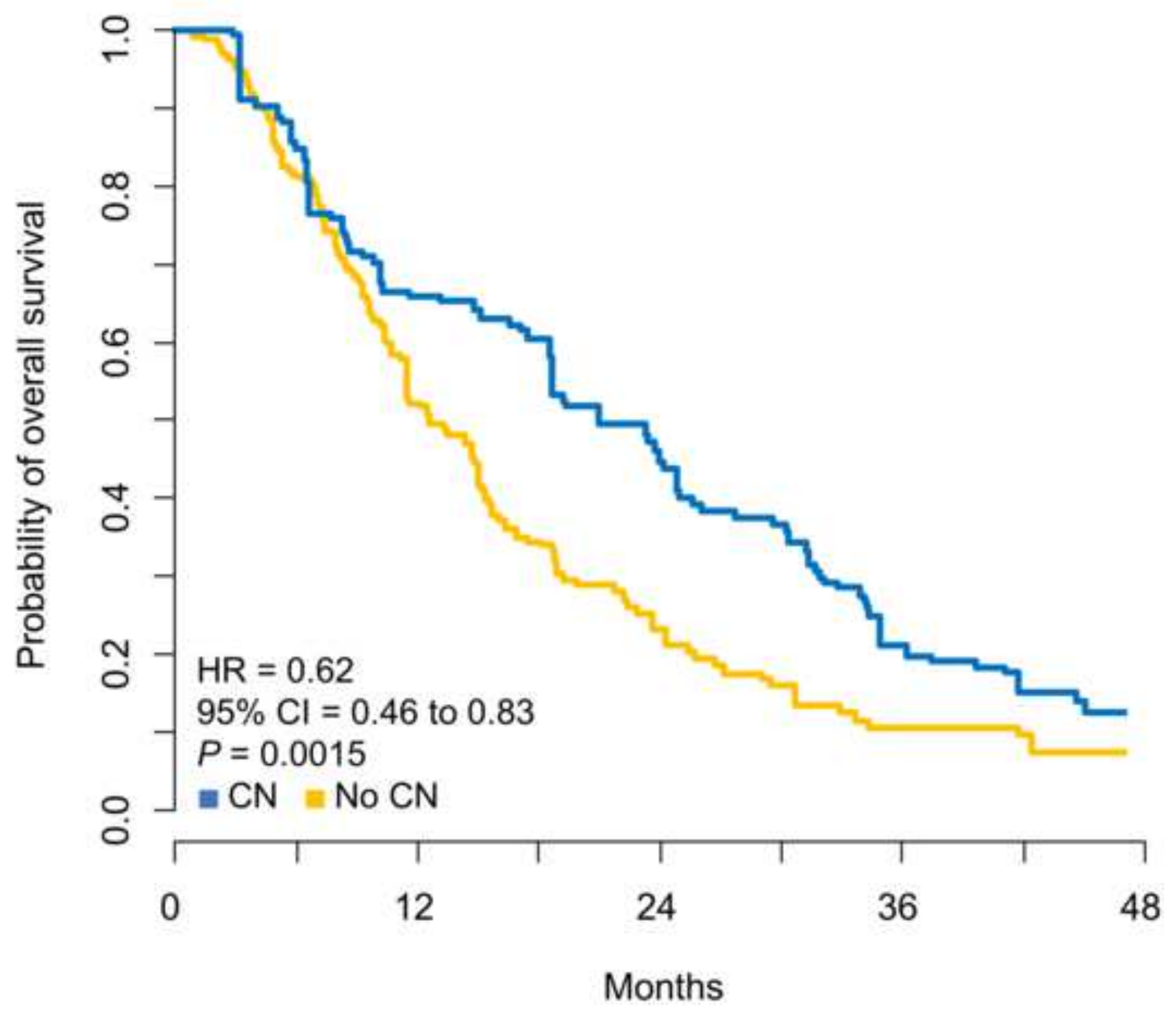




\section{Variable}

Age

Gender

Lung motastasis

Bone metastasis

Liver metastasis

Lymph node metastasis

Number of metastatic sites

\section{Subtype}

KPS

Anaemia

Neutrophilia

Hypercalcaemia

Thrombocytosis

\section{Overall}

\section{$\varepsilon=60$}

$$
>60
$$

Female

Male

Absent

Present

Absent

Present

Absent

Present

Absent

Present

2

3 or more

Clear cell

Non-clear cell

Unclassified/unknown

280

$\angle 80$

Absent

Present

Absent

Present

Absent

Present

Absent

Present

\section{HR $(95 \% \mathrm{CI})$}

$0.54(0.34-0.88)$

$0.70(0.48-1.02)$

$0.34(0.19-0.60)$

$0.77(0.54-1.09)$

$0.70(0.39-1.26)$

$0.62(0.44-0.87)$

$0.58(0.41-0.84)$

$0.77(0.46-1.31)$

$0.63(0.46-0.86)$

$0.65(0.26-1.63)$

$0.71(0.49-1.05)$

$0.49(0.31-0.79)$

$0.57(0.34-0.94)$

$0.70(0.45-1.10)$

$0.62(0.31-1.24)$

$0.64(0.46-0.89)$

$0.54(0.26-1.11)$

$1.13(0.54-2.42)$

$0.50(0.34-0.75)$

$0.95(0.61-1.48)$

$0.53(0.33-0.83)$

$0.70(0.48-1.04)$

$0.57(0.41-0.81)$

$0.90(0.51-1.62)$

$0.68(0.50-0.94)$

$0.31(0.12-0.80)$

$0.76(0.55-1.05)$

$0.25(0.12-0.54)$

$0.63(0.55-0.97)$

\section{$\boldsymbol{P}$ interaction}

0.32

0.03

0.78

0.40

0.82

0.17

0.80

0.81

0.06

0.47

0.56

0.18

0.01

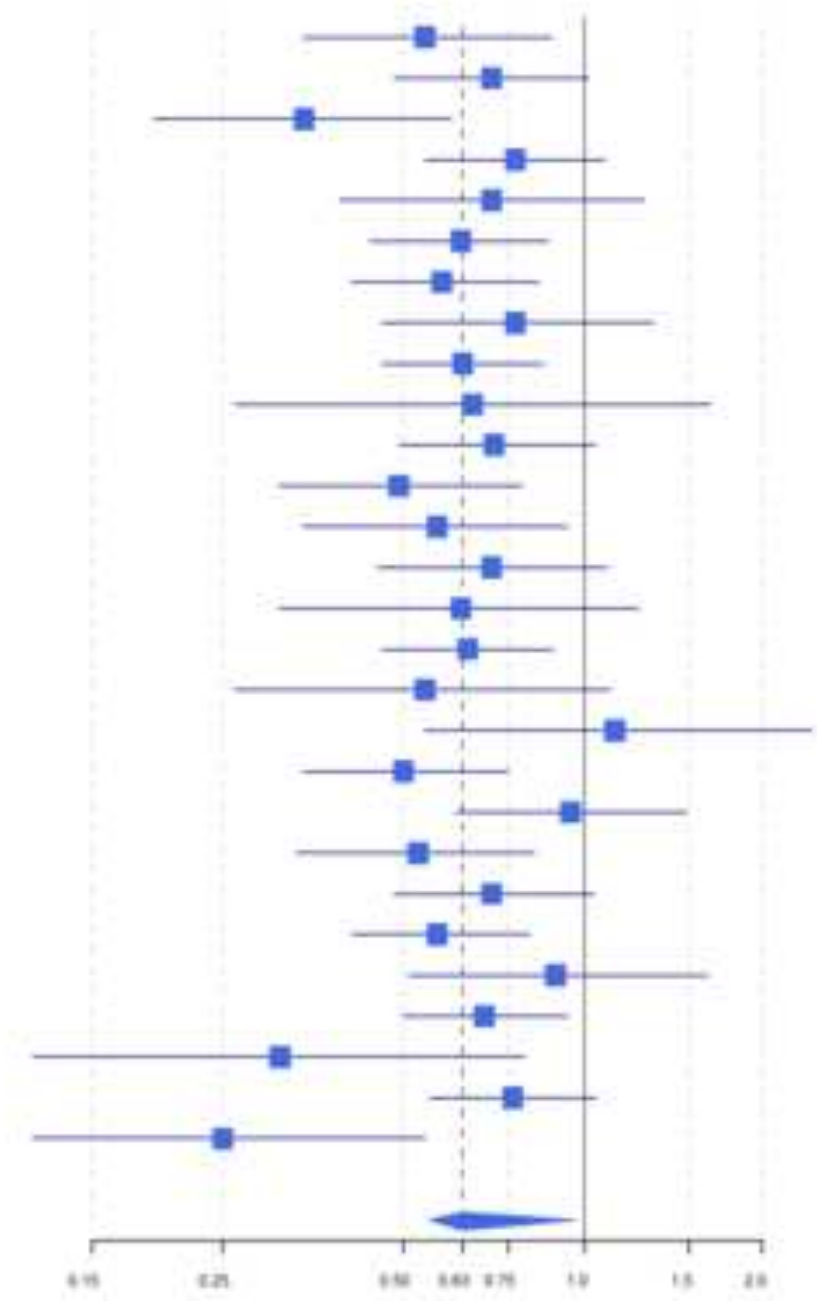




\section{Table 1}

Baseline characteristics for 261 patients who received targeted therapies with $(\mathrm{CN})$ or without cytoreductive nephrectomy (NCN) for synchronous metastatic renal cell carcinoma in the unweighted and weighted cohort of the Cambridge Oncology Registry. TT, targeted therapy.

\begin{tabular}{|c|c|c|c|c|c|c|c|}
\hline & \multicolumn{4}{|c|}{ Unweighted cohort } & \multicolumn{3}{|c|}{ IPTW-weighted cohort } \\
\hline & Overall & No CN & $\mathrm{CN}$ & $\begin{array}{c}\text { Std } \\
\text { difference }\end{array}$ & No CN & CN & $\begin{array}{c}\text { Std } \\
\text { difference }\end{array}$ \\
\hline Number of patients - $\mathrm{n}(\%)$ & $261(100)$ & $164(62.8)$ & $97(37.2)$ & - & - & - & - \\
\hline Age - mean (SD) & $62.2(11.2)$ & $64.4(9.9)$ & $58.6(12.4)$ & 0.512 & $62.36(9.39)$ & $62.2(9.65)$ & 0.017 \\
\hline Male gender - n (\%) & $181(69.3)$ & $116(70.7)$ & $65(67.0)$ & 0.08 & $44.9(69.1)$ & $44.4(68.1)$ & 0.023 \\
\hline Lung metastasis - $\mathrm{n}(\%)$ & $196(75.1)$ & $124(75.6)$ & $72(74.2)$ & 0.032 & $46.8(72.0)$ & $48.6(74.5)$ & 0.057 \\
\hline Liver metastasis - $\mathrm{n}(\%)$ & $34(13.0)$ & $25(15.2)$ & $9(9.3)$ & 0.183 & $6.5(10.0)$ & $7.5(11.5)$ & 0.048 \\
\hline Brain metastasis - $\mathrm{n}(\%)$ & $10(3.8)$ & $9(5.5)$ & $1(1.0)$ & 0.253 & $1.1(1.7)$ & $1(1.5)$ & 0.015 \\
\hline Node metastasis - $\mathrm{n}(\%)$ & $107(41.0)$ & $75(45.7)$ & $32(33.0)$ & 0.263 & $24.1(37.1)$ & $24.5(37.6)$ & 0.01 \\
\hline Bone metastasis - $\mathrm{n}(\%)$ & $89(34.1)$ & $69(42.1)$ & $20(20.6)$ & 0.475 & $17.5(26.9)$ & $16.4(25.1)$ & 0.041 \\
\hline $\begin{array}{l}\text { Number of metastatic sites }-\mathrm{n}(\%) \\
\text { One } \\
\text { Two } \\
\text { Three or more }\end{array}$ & $\begin{array}{c}97(37.2) \\
103(39.5) \\
61(23.4)\end{array}$ & $\begin{array}{l}44(26.8) \\
67(40.9) \\
53(32.3)\end{array}$ & $\begin{array}{c}53(54.6) \\
36(37.1) \\
8(8.2)\end{array}$ & 0.738 & $\begin{array}{c}30(46.3) \\
27.5(42.3) \\
7.4(11.4)\end{array}$ & $\begin{array}{c}29.3(45.0) \\
27.9(42.8) \\
8(12.3)\end{array}$ & 0.032 \\
\hline $\begin{array}{l}\text { Subtype }-\mathrm{n}(\%) \\
\text { Clear cell } \\
\text { Non-clear cell } \\
\text { Unclassified/unknown } \\
\end{array}$ & $\begin{array}{c}203(77.8) \\
39(14.9) \\
19(7.3) \\
\end{array}$ & $\begin{array}{c}123(75.0) \\
24(14.6) \\
17(10.4) \\
\end{array}$ & $\begin{array}{c}80(82.5) \\
15(15.5) \\
2(2.1) \\
\end{array}$ & 0.349 & $\begin{array}{c}53.7(82.7) \\
9.7(14.9) \\
1.6(2.4) \\
\end{array}$ & $\begin{array}{c}53.6(82.2) \\
9.6(14.7) \\
2(3.1)\end{array}$ & 0.041 \\
\hline Anaemia - n (\%) & $141(54.0)$ & $103(62.8)$ & $38(39.2)$ & 0.486 & $30(46.2)$ & $29.3(44.9)$ & 0.027 \\
\hline Neutrophilia - n (\%) & $62(23.8)$ & $49(29.9)$ & $13(13.4)$ & 0.408 & $10.8(16.6)$ & $11.5(17.6)$ & 0.028 \\
\hline Hypercalcaemia - n (\%) & $47(18.0)$ & $33(20.1)$ & $14(14.4)$ & 0.151 & $9.6(14.8)$ & $11.1(17.0)$ & 0.06 \\
\hline Thrombocytosis - $\mathrm{n}(\%)$ & $68(26.1)$ & $51(31.1)$ & $17(17.5)$ & 0.32 & $13.2(20.3)$ & $14(21.5)$ & 0.029 \\
\hline Interval to TT $<12$ months $-\mathrm{n}(\%)$ & $245(93.9)$ & $158(96.3)$ & $87(89.7)$ & 0.263 & $61(93.8)$ & $59.6(91.4)$ & 0.092 \\
\hline KPS $<80 \%-\mathrm{n}(\%)$ & $84(32.2)$ & $62(37.8)$ & $22(22.7)$ & 0.334 & $18.7(28.7)$ & $19.6(30.0)$ & 0.027 \\
\hline $\begin{array}{l}\text { IMDC prognostic group - } \mathrm{n}(\%) \\
\text { Favourable } \\
\text { Intermediate } \\
\text { Poor }\end{array}$ & $\begin{array}{c}6(2.3) \\
134(51.3) \\
121(46.4)\end{array}$ & $\begin{array}{c}1(0.6) \\
73(44.5) \\
90(54.9)\end{array}$ & $\begin{array}{c}5(5.2) \\
61(62.9) \\
31(32.0) \\
\end{array}$ & 0.522 & $\begin{array}{c}1(1.5) \\
39.7(61.1) \\
24.3(37.4)\end{array}$ & $\begin{array}{c}1.5(2.3) \\
37.3(57.2) \\
26.4(40.5)\end{array}$ & 0.092 \\
\hline
\end{tabular}




\section{Table 2}

Magnitudes of the joint bounding factor for various combinations of the odds of receiving cytoreductive nephrectomy and the hazard of overall mortality in the presence of unmeasured confounders. Blue, gold, and red areas correspond to a joint bounding factor correspond to significant, nonsignificant, and opposite treatment effect of cytoreductive nephrectomy.

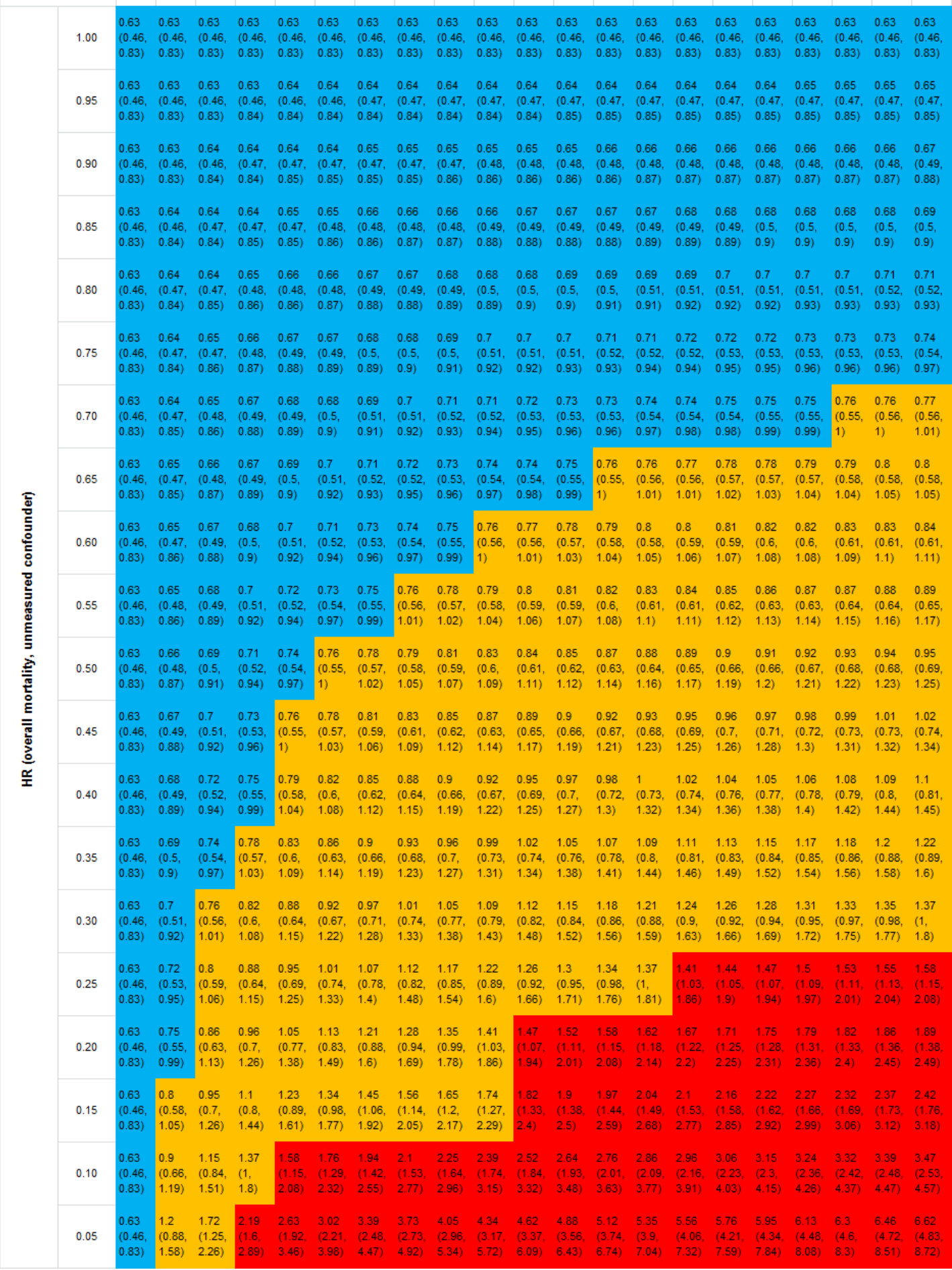




\section{Table 3}

Multivariable Cox proportional hazards model predicting overall mortality.

\begin{tabular}{lccr}
\hline Variable & HR & 95\% Cl & $\boldsymbol{P}$ value \\
\hline Age & 1.00 & $0.99-1.02$ & 0.56 \\
Male gender & 0.87 & $0.64-1.19$ & 0.4 \\
Lung metastasis & 0.97 & $0.66-1.42$ & 0.87 \\
Bone metastasis & 1.28 & $0.89-1.85$ & 0.19 \\
Liver metastasis & 1.73 & $1.13-2.64$ & 0.012 \\
Lymph node metastasis & 1.57 & $1.14-2.15$ & 0.006 \\
Brain metastasis & 0.88 & $0.39-2.00$ & 0.77 \\
Anaemia & 1.33 & $0.98-1.82$ & 0.07 \\
Hypercalcaemia & 0.82 & $0.55-1.22$ & 0.34 \\
Thrombocytosis & 1.54 & $1.08-2.20$ & 0.016 \\
KPS <80 & 1.71 & $1.24-2.37$ & 0.001 \\
Interval to TT >12 months & 1.71 & $0.93-3.16$ & 0.087 \\
Subtype & & & \\
$\quad$ Clear cell & 1.00 & & \\
$\quad$ Non-clear cell & 1.45 & $0.96-2.20$ & 0.074 \\
$\quad$ Unclassified/unknown & 0.82 & $0.45-1.48$ & 0.51 \\
Advanced line treatment (>first line) & & & \\
$\quad$ No & 1.00 & & 0.002 \\
$\quad$ Yes, not nivolumab/cabozantinib & 0.60 & $0.44-0.83$ & 0.001 \\
$\quad$ Yes, nivolumab/cabozantinib & 0.24 & $0.10-0.55$ & $<3$ \\
Cytoreductive nephrectomy & 0.68 & $0.48-0.99$ & 0.043 \\
\hline
\end{tabular}

